\title{
Resolving a Discrepancy in Diffusion Potentials, with a Case Study for Li-Ion Batteries
}

\author{
Adrien M. Bizeray, ${ }^{*}$ David A. Howey, ${ }^{* *}$ and Charles W. Monroe ${ }^{* *, \mathrm{z}}$ \\ Department of Engineering Science, University of Oxford, Oxford OX1 3PJ, United Kingdom
}

\begin{abstract}
Overpotentials induced by liquid-phase composition variation can be important when electrochemical devices are operated at high current. The dominant models that describe such 'diffusion potentials' are Nernst-Planck (dilute-solution) theory and OnsagerStefan-Maxwell (concentrated-solution) theory. Nernst-Planck flux laws emerge from Onsager-Stefan-Maxwell laws in the limit of high electrolyte dilution, and the material properties involved come into agreement. The two models yield different expressions for diffusion potentials in the dilute limit, however, because of a disparity in how electric potential is defined. As applied to lithiumion batteries, concentrated-solution theory employs a voltage measured by a reference electrode reversible to lithium cations; this provides an unambiguous connection to a measurement process, albeit hypothetical on a local scale. After the Nernst-Planck voltage is related to such a properly referenced voltage, the discrepancy in diffusion potentials vanishes. The impact of using Nernst-Planck voltages instead of measurable voltages is illustrated by simulations of a lithium-ion battery. Terminal-to-terminal voltage is relatively unaffected, but the thermal response and internal states change significantly.

(C) The Author(s) 2016. Published by ECS. This is an open access article distributed under the terms of the Creative Commons Attribution 4.0 License (CC BY, http://creativecommons.org/licenses/by/4.0/), which permits unrestricted reuse of the work in any medium, provided the original work is properly cited. [DOI: 10.1149/2.0451608jes] All rights reserved.
\end{abstract}

Manuscript received April 22, 2016. Published May 25, 2016.

In a recent paper, Ramos highlighted an observation that the electrochemical modeling community uses disparate conventions to account for the effects of concentration variation on voltage drops within liquid-electrolyte phases (Ref. 1, Remark 2). Voltages associated with concentration differences arise from the Nernstian dependence of solution free energy on composition. These 'diffusion potentials' — also known as 'concentration overpotentials' — correct the traditional form of Ohm's law for charge flux. Owing to transport processes, a prefactor involving a cation transference number $t_{+}$multiplies the term in the modified form of Ohm's law that accounts for the diffusion potential, which is known as the 'MacInnes equation'. Most sources use $2\left(1-t_{+}\right)$for this prefactor, ${ }^{2-7}$ but some use $\left(1-2 t_{+}\right) \cdot{ }^{1,8-10}$ The different factors arise from the use of distinct transport formalisms when deriving the MacInnes equation. Nernst-Planck (dilute-solution) theory yields a prefactor of $\left(1-2 t_{+}\right)$in the diffusion-potential term; Onsager-Stefan-Maxwell (concentrated-solution) theory instead suggests a factor of $2\left(1-t_{+}\right)$.

This paper aims to explain the apparent disagreement between the Nernst-Planck (NP) and Onsager-Stefan-Maxwell (OSM) MacInnes equations, which is resolved by considering how electric potential is defined in the two transport theories. The discrepancy is primarily of a semantic nature, arising from ambiguity in how species electrochemical potentials should be determined from NP theory. It is shown that the NP potential arises in a natural way, as the quasi-electrostatic potential associated with an ion in a dilute, ideal solution. One of the main objectives of this paper is to explain how to relate this potential to species electrochemical potentials - and consequently, to voltages measured by reference electrodes - in a principled way.

In dilute-solution theory, the voltage represents an electric potential within a solution; since electrolytic solutions contain no free electrons, such a potential is not directly measurable - even in principle - and is therefore in some sense fictitious. In concentrated-solution theory, the voltage is explicitly defined as being a potential measured by a reference electrode of a given kind, i.e., the potential energy obtained by electrons in a reactive, electronically conductive electrode at open circuit when its surface is equilibrated with redox-active species in an adjacent solution phase. When the NP voltage is made thermodynamically consistent by being associated with an experimental measurement protocol similar to the OSM voltage, the diffusion potentials predicted by both theories come into agreement.

Developments of the two transport theories are reiterated to illustrate how the difference in MacInnes equations arises. Model

\footnotetext{
*Electrochemical Society Student Member

***Electrochemical Society Member.

${ }^{\mathrm{z} E}$ E-mail: charles.monroe@eng.ox.ac.uk
}

equations applicable to locally electroneutral solutions containing dissolved simple salts are produced. The conditions that reveal dilutesolution theory to be a limiting case of concentrated-solution theory are also laid out in detail.

In a closing section, a case study using a standard model of a lithium-ion 18650 cell is presented. Simulation results highlight the effect of using the experimentally inaccessible NP voltage $\Phi_{\mathrm{NP}}$ instead of the measurable potential $\Phi$. Although the use of $\Phi_{\mathrm{NP}}$ in place of $\Phi$ has a minimal impact on predicted cell voltage and discharge capacity, its effect on temperature rise is shown to be substantial at high discharge rates.

\section{General Considerations}

A 'binary electrolytic solution of a binary electrolyte' is a locally homogeneous phase comprising one type of cation, one type of anion, and a neutral solvent, whose properties are labeled with subscripts + , - , and 0 , respectively; these species are assumed to have equivalent charges $z_{+}>0, z_{-}<0$, and $z_{0}=0$.

The two ions compose a salt that balances charge if isolated from the solution. Ion charges are therefore constrained by the Guggenheim relation

$$
z_{+} \nu_{+}+z_{-} \nu_{-}=0,
$$

where $\nu_{+}$and $\nu_{-}$represent the ion stoichiometries in a formula unit of salt, which is taken to contain $v=v_{+}+v_{-}$ions in total. On the grounds that simulation volume elements are large in comparison to the Debye screening length, one can assume that the molar species concentrations in solution $c_{i}$ obey a local electroneutrality relationship,

$$
\sum_{i} z_{i} c_{i}=0 \quad \text { or } \quad z_{+} c_{+}+z_{-} c_{-}=0 .
$$

The second form follows because the solvent is neutral. Owing to Equation 2, either ion concentration can be described in terms of the concentration $c$ of neutral-salt formula units, since Guggenheim condition 1 implies that

$$
c=\frac{c_{+}}{v_{+}}=\frac{c_{-}}{v_{-}} .
$$

Note that Newman ${ }^{11}$ uses relationships $1-3$ freely in his manipulation of transport equations, usually without specific mention.

A last general equation defines the ionic current density $\vec{i}$. This relates to the molar species fluxes $\vec{N}_{i}$ through Faraday's law,

$$
\vec{i}=F \sum_{i} z_{i} \vec{N}_{i}=F\left(z_{+} \vec{N}_{+}+z_{-} \vec{N}_{-}\right),
$$


in which $F$ stands for Faraday's constant.

\section{Dilute-Solution Transport}

Ion-flux and current/field relations. - The NP dilute-solution theory is a starting point for many analyses of electrochemical transport phenomena; its current formulation differs little from the original statement by Planck. ${ }^{12}$ In the constitutive laws for flux, species diffusion is taken to be driven by gradients in ion concentration, while migration of charged species is driven by the electric field $\vec{E}$, and convection is driven by the bulk-flow velocity $\vec{v}$, through

$$
\begin{aligned}
& \vec{N}_{+}=-D_{+} \vec{\nabla} c_{+}+z_{+} c_{+} u_{+} F \vec{E}+c_{+} \vec{v} \\
& \vec{N}_{-}=-D_{-} \vec{\nabla} c_{-}+z_{-} c_{-} u_{-} F \vec{E}+c_{-} \vec{v} .
\end{aligned}
$$

Here $D_{i}$ is the diffusivity of species $i$, and $u_{i}$, its ionic mobility. Dilute ions also obey the Nernst-Einstein relation

$$
D_{i} \approx u_{i} R T
$$

where $R$ is the gas constant and $T$ the absolute temperature; this reduces the number of independent transport properties to two. (Onsager's transport theory ${ }^{13}$ shows that the NP model either overspecifies or underspecifies transport properties-see below.) Under the NernstEinstein approximation, the ion-flux laws become

$$
\begin{aligned}
& \vec{N}_{+}=-D_{+} \vec{\nabla} c_{+}+D_{+} c_{+} \frac{F z_{+} \vec{E}}{R T}+c_{+} \vec{v} \\
& \vec{N}_{-}=-D_{-} \vec{\nabla} c_{-}+D_{-} c_{-} \frac{F z_{-} \vec{E}}{R T}+c_{-} \vec{v} .
\end{aligned}
$$

Note that $F \vec{E} / R T$ has dimensions of inverse length.

When simulating electrolytes, one does not use the NP equations in their elementary forms, because the properties involved are not directly measurable. One instead exchanges the fundamental transport coefficients $D_{i}$ (or $D_{i}$ and $u_{i}$ ) for three macroscopically observable quantities: the ionic conductivity $\kappa$, the Fickian diffusivity $D$, and the cation transference number $t_{+}$. These properties relate to the two $D_{i}$ as follows.

Substitute the simplified NP laws into Faraday's law, then apply electroneutrality; the convection term vanishes. After introducing $c$ from Equation 3, then using the Guggenheim condition to make the substitution $z_{-} \nu_{-}=-z_{+} \nu_{+}$, one retrieves

$$
\vec{i}=-\frac{F^{2} z_{+} z_{-} c\left(\nu_{-} D_{+}+\nu_{+} D_{-}\right)}{R T} \vec{E}-F z_{+} \nu_{+}\left(D_{+}-D_{-}\right) \nabla c .
$$

Here the coefficient of the electric field must identify with the ionic conductivity,

$$
\kappa=-\frac{F^{2} z_{+} z_{-}\left(\nu_{-} D_{+}+\nu_{+} D_{-}\right) c}{R T},
$$

so that the current flux reduces to Ohm's law $\vec{i}=\kappa \vec{E}$ in the absence of concentration gradients. Generally, a current-voltage relation that expresses Ohm's law with a correction for composition gradients is called a 'MacInnes equation', although MacInnes derived his relationship by thermodynamic considerations,${ }^{14}$ rather than transport theory.

The modified form of Ohm's law allows elimination of the electric field from the NP laws in favor of the current density. Making that substitution gives

$$
\begin{aligned}
& \vec{N}_{+}=-v_{+}\left(\frac{v D_{+} D_{-}}{\nu_{-} D_{+}+\nu_{+} D_{-}}\right) \vec{\nabla} c+\left(\frac{\nu_{-} D_{+}}{\nu_{-} D_{+}+\nu_{+} D_{-}}\right) \frac{\vec{i}}{F z_{+}}+\nu_{+} c \vec{v} \\
& \vec{N}_{-}=-\nu_{-}\left(\frac{v D_{+} D_{-}}{\nu_{-} D_{+}+\nu_{+} D_{-}}\right) \vec{\nabla} c+\left(\frac{\nu_{+} D_{-}}{\nu_{-} D_{+}+\nu_{+} D_{-}}\right) \frac{\vec{i}}{F z_{-}}+\nu_{-} c \vec{v} .
\end{aligned}
$$

Inspection of these laws allows one to identify the salt diffusivity and cation transference number,

$$
\begin{aligned}
& D=\frac{\nu D_{+} D_{-}}{\nu_{-} D_{+}+\nu_{+} D_{-}}=\frac{\left(z_{+}-z_{-}\right) D_{+} D_{-}}{z_{+} D_{+}-z_{-} D_{-}} \text {and } \\
& t_{+}=\frac{\nu_{-} D_{+}}{\nu_{-} D_{+}+\nu_{+} D_{-}}=\frac{z_{+} D_{+}}{z_{+} D_{+}-z_{-} D_{-}}
\end{aligned}
$$

Summary of Nernst-Planck equations.-As a result of the definitions of $D$ and $t_{+}$in Equation 11, the NP theory yields ion-flux laws

$$
\begin{aligned}
& \vec{N}_{+}=-v_{+} D \vec{\nabla} c+t_{+} \frac{\vec{i}}{F z_{+}}+v_{+} c \vec{v} \\
& \vec{N}_{-}=-v_{-} D \vec{\nabla} c+\left(1-t_{+}\right) \frac{\vec{i}}{F z_{-}}+v_{-} c \vec{v} .
\end{aligned}
$$

The MacInnes current/field relation can be expressed as

$$
\vec{i}=\kappa \vec{E}+\frac{F z_{+} \nu_{+} D\left(\nu_{-}-v t_{+}\right)}{\nu t_{+}\left(1-t_{+}\right)} \vec{\nabla} c,
$$

after one replaces the coefficient of the field with the ionic conductivity defined in Equation 9, then uses the salt diffusivity and transference number from Equation 11 to eliminate ion diffusivities from the diffusion-potential term. One way to define a 'quasi-electrostatic potential $^{15}$ is to identify $\vec{E}$ with $-\vec{\nabla} \Phi_{\mathrm{NP}}$ here. Some additional thermodynamic restrictions are needed (cf. the section focused on the Discrepancy and its resolution) to make it consistent with Smyrl and Newman's definition of a quasi-electrostatic potential, ${ }^{15}$ however, so we might instead call $\Phi_{\mathrm{NP}}$ the Nernst-Planck voltage, explaining its subscript.

A deficiency of NP theory can be clarified by considering the definitions used to derive Equations 12 and 13. Equations 11 imply that $D_{+}=v_{+} D / v\left(1-t_{+}\right)$and $D_{-}=v_{-} D / v t_{+}$, so the ionic conductivity in Equation 9 should also depend on $D$ and $t_{+}$according to

$$
\kappa=\frac{F^{2} z_{+}^{2} \nu_{+}^{2} D c}{\nu R T t_{+}\left(1-t_{+}\right)} .
$$

Table I compares the conductivity predicted from experimental values of $D$ and $t_{+}$via Equation 14 to the measured ionic conductivity for several aqueous electrolytes at various concentrations. Relation 14 holds well at $5 \mathrm{mM}$ for strong acids and bases - but even for simple buffers, its error at this dilution ranges from $1 \%-4 \%$. This error also increases rapidly with concentration in all cases. At $1 \mathrm{M}$, Equation 14 is in error by $25 \%-58 \%$, depending on the electrolyte in question.

Thus the relationship among $D, t_{+}$, and $\kappa$ suggested by NP theory is not borne out by experiments, even at relatively high dilution. Nevertheless, Equation 14 is often used to cast Equation 13 in the alternate form

$$
\frac{\vec{i}}{\kappa}=-\vec{\nabla} \Phi_{\mathrm{NP}}+\frac{R T\left(\nu_{-}-\nu t_{+}\right)}{F z_{+} \nu_{+}} \vec{\nabla} \ln c,
$$

which will be useful further along in the discussion.

\section{Concentrated-Solution Transport}

The Onsager-Stefan-Maxwell formalism.-OSM transport theory is based on principles of irreversible thermodynamics; ${ }^{16}$ its application to electrolytes was developed significantly by Newman. ${ }^{17-19}$ In concentrated-solution theory, thermodynamically consistent transport laws are formulated by linearly relating a set of bulk-velocityindependent species fluxes to a set of thermodynamically independent driving forces.

One identifies the basis sets of fluxes and forces by deriving an expression that describes the instantaneous local entropy generation (energy dissipation) implied by the differential balances of material, momentum, and energy. This process leads to a conclusion that the 


\begin{tabular}{|c|c|c|c|c|}
\hline Solute & $\begin{array}{l}\text { Conc. } \\
{[\mathrm{M}]}\end{array}$ & $\begin{array}{c}\kappa_{\text {thry }} \\
{\left[\mathrm{mScm}^{-1}\right]}\end{array}$ & $\begin{array}{c}\kappa_{\text {expt }} \\
{\left[\mathrm{mScm}^{-1}\right]}\end{array}$ & $\begin{array}{c}\text { Error } \\
{[\%]}\end{array}$ \\
\hline \multirow[t]{5}{*}{$\mathrm{HCl}^{\mathrm{a}}$} & 0.005 & 2.080 & 2.078 & 0.1 \\
\hline & 0.01 & 4.126 & 4.118 & 0.2 \\
\hline & 0.1 & 40.42 & 39.12 & 3.3 \\
\hline & 0.5 & 220.7 & 180.4 & 22.3 \\
\hline & 1.0 & 492.0 & 333.6 & 47.5 \\
\hline \multirow[t]{5}{*}{$\mathrm{NaOH}$} & 0.005 & 1.210 & 1.207 & 0.3 \\
\hline & 0.01 & 2.415 & 2.385 & 1.3 \\
\hline & 0.1 & 24.04 & 21.76 & 10.5 \\
\hline & 0.5 & 120.2 & 98.48 & 22.0 \\
\hline & 1.0 & 240.6 & 181.3 & 32.7 \\
\hline \multirow[t]{5}{*}{$\mathrm{NaCl}$} & 0.005 & 0.614 & 0.603 & 1.8 \\
\hline & 0.01 & 1.219 & 1.185 & 2.9 \\
\hline & 0.1 & 11.77 & 10.67 & 10.2 \\
\hline & 0.5 & 59.06 & 46.81 & 26.2 \\
\hline & 1.0 & 119.7 & 85.76 & 39.5 \\
\hline \multirow[t]{5}{*}{$\mathrm{KCl}$} & 0.005 & 0.727 & 0.718 & 1.3 \\
\hline & 0.01 & 1.441 & 1.413 & 2.0 \\
\hline & 0.1 & 13.89 & 12.90 & 7.7 \\
\hline & 0.5 & 68.95 & 57.43 & 20.0 \\
\hline & 1.0 & 141.0 & 112.0 & 25.9 \\
\hline \multirow[t]{5}{*}{$\mathrm{BaCl}_{2}{ }^{\mathrm{a}}$} & 0.005 & 1.286 & 1.239 & 3.8 \\
\hline & 0.01 & 2.534 & 2.382 & 6.4 \\
\hline & 0.1 & 23.91 & 19.96 & 19.8 \\
\hline & 0.5 & 123.2 & 87.48 & 40.9 \\
\hline & 1.0 & 258.3 & 163.0 & 58.5 \\
\hline \multirow[t]{5}{*}{$\mathrm{K}_{2} \mathrm{SO}_{4}$} & 0.005 & $1.328^{\mathrm{b}}$ & 1.340 & -0.9 \\
\hline & 0.01 & 2.624 & 2.550 & 2.9 \\
\hline & 0.1 & 24.00 & 20.23 & 18.6 \\
\hline & 0.5 & $99.80^{\mathrm{b}}$ & 81.50 & 22.5 \\
\hline & 1.0 & \multicolumn{2}{|c|}{ insoluble } & $\sim$ \\
\hline
\end{tabular}

${ }^{\text {a }}$ The transference number $t_{+}$was measured using experimental techniques that assume Equations 12 only (common to both theories), excepting for $\mathrm{BaCl}_{2}$ and $\mathrm{HCl}$, where the EMF technique was used, and therefore a MacInnes equation from OSM theory was assumed in data processing.

${ }^{\mathrm{b}}$ Extrapolated from available experimental data.

fluxes comprise excess species velocities, while the forces involve gradients of the species electrochemical potentials $\mu_{i} .{ }^{20}$ Since electrochemical potential depends on both composition and electrical state, the thermodynamic forces drive both diffusion and migration in systems where species carry electric charge.

Isothermal, isobaric binary electrolytic solutions of binary electrolytes comprise three electrochemical species. A local GibbsDuhem equation

$$
c_{+} \vec{\nabla} \mu_{+}+c_{-} \vec{\nabla} \mu_{-}+c_{0} \vec{\nabla} \mu_{0}=\overrightarrow{0}
$$

relates the species' associated diffusion driving forces everywhere in the phase, so only two of the three forces are independent. Inspection of the dissipation ${ }^{21}$ suggests that these driving forces relate to species fluxes through two independent OSM flux laws,

$$
\begin{aligned}
& -c_{0} \vec{\nabla} \mu_{0}=\frac{R T}{c_{\mathrm{T}}}\left(\frac{c_{+} \vec{N}_{0}-c_{0} \vec{N}_{+}}{\mathscr{D}_{0+}}+\frac{c_{-} \vec{N}_{0}-c_{0} \vec{N}_{-}}{\mathscr{D}_{0-}}\right) \\
& -c_{+} \vec{\nabla} \mu_{+}=\frac{R T}{c_{\mathrm{T}}}\left(\frac{c_{0} \vec{N}_{+}-c_{+} \vec{N}_{0}}{\mathscr{D}_{0+}}+\frac{c_{-} \vec{N}_{+}-c_{+} \vec{N}_{-}}{\mathscr{D}_{+-}}\right),
\end{aligned}
$$

in which the Stefan-Maxwell coefficients $\mathscr{D}_{i j}$ describe binary species/species interactions and $c_{\mathrm{T}}=c_{0}+c_{+}+c_{-}$defines the total molar concentration of the solution. Note that the Onsager reciprocal relation $\mathscr{D}_{i j}=\mathscr{D}_{j i}$ has been used to replace $\mathscr{D}_{+0}$ with $\mathscr{D}_{0+}$ in the equation for $c_{+} \vec{\nabla} \mu_{+}$.

Onsager discussed how proper transport constitutive laws should associate a single transport property with each pair of independent fluxes (or forces). For a three-species diffusion system, there are three such pairwise combinations. OSM equations thus provide a more complete description of transport than NP laws: as well as using thermodynamic diffusion driving forces, they properly incorporate three independent transport properties, rather than two (or four, if the Nernst-Einstein relation is ignored).

Any molar species flux can be expressed in terms of a species velocity $\vec{v}_{i}$ through the definition $\vec{N}_{i}=c_{i} \vec{v}_{i}$, allowing the solvent flux to be replaced with its velocity wherever it appears. After applying electroneutrality, the equation describing the solvent force becomes

$$
-\frac{c_{\mathrm{T}}}{R T} \vec{\nabla} \mu_{0}=-\frac{\vec{N}_{+}}{\mathscr{D}_{0+}}-\frac{\vec{N}_{-}}{\mathscr{D}_{0-}}+\left(\frac{\nu_{+}}{\mathscr{D}_{0+}}+\frac{\nu_{-}}{\mathscr{D}_{0-}}\right) c \vec{v}_{0} .
$$

Faraday's law can then be used to eliminate the anion flux,

$$
\begin{aligned}
\vec{N}_{+}= & \frac{\nu_{+} c_{\mathrm{T}}}{R T}\left(\frac{\mathscr{D}_{0+} \mathscr{D}_{0-}}{\nu_{-} \mathscr{D}_{0+}+\nu_{+} \mathscr{D}_{0-}}\right) \vec{\nabla} \mu_{0} \\
& +\left(\frac{\nu_{-} \mathscr{D}_{0+}}{\nu_{-} \mathscr{D}_{0+}+\nu_{+} \mathscr{D}_{0-}}\right) \frac{\vec{i}}{F z_{+}}+\nu_{+} c \vec{v}_{0},
\end{aligned}
$$

or it can be used to eliminate the cation flux, leaving

$$
\begin{aligned}
\vec{N}_{-}= & \frac{\nu_{-} c_{\mathrm{T}}}{R T}\left(\frac{\mathscr{D}_{0+} \mathscr{D}_{0-}}{\nu_{-} \mathscr{D}_{0+}+\nu_{+} \mathscr{D}_{0-}}\right) \vec{\nabla} \mu_{0} \\
& +\left(\frac{\nu_{+} \mathscr{D}_{0-}}{\nu_{-} \mathscr{D}_{0+}+\nu_{+} \mathscr{D}_{0-}}\right) \frac{\vec{i}}{F z_{-}}+\nu_{-} c \vec{v}_{0} .
\end{aligned}
$$

It is worth emphasizing that in concentrated-solution theory, both ion-flux laws derive from a single OSM force law - Equation 18 - by combination with Faraday's law. A diffusivity and transference number with definitions similar to the macroscopic NP properties arise (compare Equation 11),

$$
\begin{aligned}
\mathscr{D} & =\frac{\nu \mathscr{D}_{0+} \mathscr{D}_{0-}}{\nu_{-} \mathscr{D}_{0+}+\nu_{+} \mathscr{D}_{0-}}=\frac{\left(z_{+}-z_{-}\right) \mathscr{D}_{0+} \mathscr{D}_{0-}}{z_{+} \mathscr{D}_{0+}-z_{-} \mathscr{D}_{0-}} \text { and } \\
t_{+}^{0} & =\frac{\nu_{-} \mathscr{D}_{0+}}{\nu_{-} \mathscr{D}_{0+}+\nu_{+} \mathscr{D}_{0-}}=\frac{z_{+} \mathscr{D}_{0+}}{z_{+} \mathscr{D}_{0+}-z_{-} \mathscr{D}_{0-}} .
\end{aligned}
$$

Some fine-graining of definitions is needed here: $\mathscr{D}$ is a 'thermodynamic diffusivity', based on a chemical-potential-gradient diffusion driving force rather than a concentration-gradient driving force; and $t_{+}^{0}$ is a 'cation transference number relative to the solvent velocity', since $\vec{v}_{0}$ is the convective velocity that appears in flux laws 19 and 20 .

Thermodynamics of dissolved salts.-Thermodynamic laws can be incorporated to make the flux laws and MacInnes equation for concentrated binaries more concrete. Any salt $\mathrm{M}_{v_{+}} \mathrm{X}_{v_{-}}$in solution experiences a dissociation equilibrium,

$$
\mathrm{M}_{v_{+}} \mathrm{X}_{v_{-}} \leftrightharpoons v_{+} \mathrm{M}^{z+}+v_{-} \mathrm{X}^{z_{-}}
$$

This establishes a relationship among electrochemical potentials. If the neutral-salt chemical potential is defined as $\mu_{\mathrm{e}}$, then dissociation equilibrium requires that the ion potentials satisfy

$$
\mu_{\mathrm{e}}=v_{+} \mu_{+}+\nu_{-} \mu_{-} .
$$

Also, since electrolyte formula units have neutral charge, the thermodynamic function $\mu_{\mathrm{e}}$ is necessarily independent of electrical state (i.e., voltage). Combining equilibrium Equation 23 with Gibbs-Duhem Equation 16 for a locally electroneutral system, one finds that

$$
c_{0} \vec{\nabla} \mu_{0}=-c \vec{\nabla} \mu_{\mathrm{e}},
$$

allowing terms involving the solvent chemical-potential gradient to be expressed in terms of the electrolyte chemical-potential gradient. 
Further, one can introduce a thermodynamic factor $\chi$, which establishes how the electrolyte chemical-potential gradient relates to its concentration gradient. Following Liu and Monroe, ${ }^{22}$ the factor can be defined through

$$
c \vec{\nabla} \mu_{\mathrm{e}}=\frac{\nu R T \chi}{c_{\mathrm{T}} \bar{V}_{0}} \vec{\nabla} c .
$$

The denominator in the coefficient of the gradient on the right is an 'excluded-volume factor', which arises because local total solution concentration (or, equivalently, solvent concentration) depends on composition. ${ }^{\mathrm{a}}$

Thermodynamics of solution volume.-The excluded-volume factor can be understood in more detail by considering that as a consequence of the extensivity of volume, electroneutral binary electrolytes satisfy a local equation of state

$$
c \bar{V}_{\mathrm{e}}+c_{0} \bar{V}_{0}=1
$$

where $\bar{V}_{\mathrm{e}}=\nu_{+} \bar{V}_{+}+\nu_{-} \bar{V}_{-}$and $\bar{V}_{0}$ are the partial molar volumes of electrolyte and solvent, respectively. Since solution volume also satisfies a Gibbs-Duhem equation, the partial molar volumes in a binary electrolytic solution at fixed $T$ and $p$ relate through

$$
\left(\frac{d \bar{V}_{0}}{d c}\right)_{T, p}=-\frac{c}{1-c \bar{V}_{\mathrm{e}}}\left(\frac{d \bar{V}_{\mathrm{e}}}{d c}\right)_{T, p},
$$

and are functions of $c$. (The mass densities of many electrolytic solutions vary linearly with molar salt concentration; in such cases both partial molar volumes are constant with composition.) Equation 26 shows that the excluded-volume factor can be expressed as

$$
\bar{V}_{0} c_{\mathrm{T}}=1+\left(\nu \bar{V}_{0}-\bar{V}_{\mathrm{e}}\right) c
$$

and Equation 27 shows that the excluded-volume factor is a function of $c$ only.

The thermodynamic relations above allow $\vec{\nabla} \mu_{0}$ to be expressed in terms of $\vec{\nabla} c$ and the parameters $\bar{V}_{0}, \bar{V}_{\mathrm{e}}$, and $\chi$, which all depend at most on $c$ at constant $T$ and $p$. Inserting Equations 24 through 26 and 28 into Equations 19 and 20 yields constitutive laws for the ion fluxes that compare quite closely to Equations 12 . By comparison one can cast the Fickian diffusivity in terms of OSM properties,

$$
D=\frac{\mathscr{D} \chi}{1-c \bar{V}_{\mathrm{e}}} \text {. }
$$

Thus thermodynamic and excluded-volume corrections are both convolved into Fickian diffusivities. This convolution may help explain why the concentration and temperature dependences of $D$ vary so widely among different electrolytic solutions, even in cases where the same solvent is used.

Thermodynamics of reference electrodes.-Because electrochemical potentials are used in the fundamental transport laws, the identification of the electric field in OSM theory is less straightforward than in NP theory. To have thermodynamic meaning, voltage

a Several composition bases can be used equivalently. In the notation of Newman and Thomas-Alyea, ${ }^{11} \chi$ relates to the mean molar activity coefficient of the salt on a molar basis, $f_{+-}$:

$$
\chi=c_{\mathrm{T}} \bar{V}_{0}\left[1+\left(\frac{d \ln f_{+-}}{d \ln c}\right)_{T, p}\right]=1+\left(\frac{d \ln \lambda_{+-}}{d \ln y}\right)_{T, p} .
$$

The far right equality shows that $\chi$ also relates very simply to the derivative of the mean molar salt activity coefficient $\lambda_{+-}$over a 'particle-fraction basis', in which $y=c / c_{\mathrm{T}}$ describes salt content.

Particle fractions may be more natural to express the composition dependences of thermodynamic factors because species activities based on them satisfy the Gibbs-Duhem equation naturally in the ideal-solution limit, where all activity coefficients approach 1 . Further benefits are that the relationship between $\chi$ and $\lambda_{+-}$requires no separate excludedvolume factor, and, perhaps most importantly, $y$ can easily be varied independently of $T$ and $p$. must be measured using a 'reference electrode of a given kind', ${ }^{24}$ reversible to one, some, or all of the species in the solution phase.

Since our objective is to clarify the $1-t_{+}$vs. $1-2 t_{+}$discrepancy pointed out by Ramos, ${ }^{1}$ it will be assumed that there is a reference electrode reversible only to cations, as is typical for lithium-ion battery systems. In that case, one imagines that the cation undergoes complete reduction,

$$
s_{+} \mathrm{M}^{z+}+n \mathrm{e}^{-} \leftrightharpoons \mathrm{M}^{0},
$$

to form a distinct phase comprising a metal $\mathrm{M}^{0}$; further one supposes that an electrical contact can be made to this metal, allowing measurement of the energy of conduction-band electrons $\mathrm{e}^{-}$within it. (A charge balance requires that if the cation reaction stoichiometry is $s_{+}$, then the number of electrons $n$ satisfies $s_{+} z_{+}+n z_{\mathrm{e}}^{-}=0$, where $z_{\mathrm{e}^{-}}=-1$ is the electron's equivalent charge.) A voltmeter actually measures the difference between the electrochemical potentials of electrons in two electrodes, rather than measuring $\mu_{+}$directly. But if the electrodes are reference electrodes, then these quantities relate, because the half-reaction equilibrium requires that

$$
s_{+} \mu_{+}+n \mu_{\mathrm{e}^{-}}=\mu_{\mathrm{M}^{0}} .
$$

The pure metal $\mathbf{M}^{0}$ forms an isolated phase, so the associated chemical potential depends only on $T$ and $p$. The electron's electrochemical potential is related to the measured voltage through a constitutive law

$$
\mu_{\mathrm{e}^{-}}=\mu_{\mathrm{e}^{-}}^{\ominus}+F z_{\mathrm{e}^{-}} \Phi
$$

where $\mu_{\mathrm{e}^{-}}^{\ominus}$ is the Fermi level of the metal. Since the metal's Fermi level is independent of electrolyte composition, a gradient of Equation 31 yields $^{\mathrm{b}}$

$$
\frac{n z_{\mathrm{e}^{-}}}{z_{+}}\left(-\vec{\nabla} \mu_{+}+F z_{+} \vec{\nabla} \Phi\right)=\overrightarrow{0}, \quad \text { or } \quad \vec{\nabla} \mu_{+}=F z_{+} \vec{\nabla} \Phi
$$

So if a series of pointwise measurements with a metallic reference electrode that can implement complete reduction of the cation is used to determine a potential gradient, said gradient will be directly proportional to the gradient of cation electrochemical potential in the solution.

Insertion of Equation 33 yields a MacInnes relation comparable to Equation 15, in which the ionic conductivity can be identified in the absence of concentration gradients as

$$
\frac{1}{\kappa}=\frac{R T}{F^{2} z_{+}^{2} \nu_{+}^{2} c_{\mathrm{T}}}\left[\frac{\nu_{+} \nu_{-}}{\mathscr{D}_{+-}}+\frac{\nu c_{0} t_{+}^{0}\left(1-t_{+}^{0}\right)}{\mathscr{D} c}\right] .
$$

This definition involves the third transport coefficient $\mathscr{D}_{+-}$. Thus $\kappa$ is independently variable if $\mathscr{D}$ and $t_{+}^{0}$ are both fixed, and the problem illustrated by Table I can be resolved.

Summary of OSM equations.-For binary electrolytes OSM theory yields ion-flux laws in the form

$$
\begin{aligned}
& \vec{N}_{+}=-v_{+} D \vec{\nabla} c+t_{+}^{0} \frac{\vec{i}}{F z_{+}}+v_{+} c \vec{v}_{0}, \\
& \vec{N}_{-}=-v_{-} D \vec{\nabla} c+\left(1-t_{+}^{0}\right) \frac{\vec{i}}{F z_{-}}+v_{-} c \vec{v}_{0},
\end{aligned}
$$

\footnotetext{
${ }^{b}$ Differentiating Equation 31 is an informal step taken for brevity; one might take issue because Equation 31 holds across a surface, rather than within a volume (as Equation 33). A more formally rigorous approach is to express the change in cation electrochemical potential between two points within the liquid phase, $\Delta \mu_{+}$, as a line integral of $\vec{\nabla} \mu_{+}$ through the liquid between those points (any connecting path yields the same $\Delta \mu_{+}$since $\mu_{+}$is a state function); then $\mu_{+}$values at the termini can be related to $\mu_{\mathrm{e}^{-}}$values within hypothetical reference electrodes placed there, through interfacial half-reaction equilibria in the form of Equation 31. The rigorous process (line integration followed by application of electrochemical equilibria at terminal points) has been carried out in detail by the authors elsewhere, ${ }^{22,23}$ and yields identical results after many more intermediate steps. The gradient approach has also been employed by many others, most notably by Newman and Thomas-Alyea, ${ }^{11}$ who use it in the chapter focused on concentrated-solution theory, but who also discuss the equivalent line-integral approach at length in an earlier chapter focused on the thermodynamics of cells with liquid junctions.
} 
which have been simplified from Equations 19 and 20 by incorporating Equation 24 and the definition of $\chi$ from Equation 25, making the replacements $\mathscr{D}_{0+}=v_{+} \mathscr{D} / \nu\left(1-t_{+}^{0}\right)$ and $\mathscr{D}_{0-}=v_{+} \mathscr{D} / \nu t_{+}^{0}$ through Equations 21, using Equations 26 and 28 to eliminate $c_{0}$ and $c_{\mathrm{T}}$ in favor of $c$, and finally introducing the Fickian diffusivity from Equation 29.

If one measures the electric potential by using a reference electrode with which only cations react, and at which cations completely reduce into a separate pure solid phase, one retrieves the MacInnes equation

$$
\frac{\vec{i}}{\kappa}=-\vec{\nabla} \Phi+\frac{R T \nu\left(1-t_{+}^{0}\right) \chi}{F z_{+} \nu_{+} c_{\mathrm{T}} \bar{V}_{0}} \vec{\nabla} \ln c .
$$

This is quite similar to Equation 15, save for the prefactor in the diffusion potential.

\section{The Discrepancy and Its Resolution}

Given a solution of a 1:1 electrolyte like $\mathrm{LiPF}_{6}$, if a Li-metal reference is used, then MacInnes Equation 36, which involves the reference-electrode potential $\Phi$, has a term $2\left(1-t_{+}^{0}\right)$ in the numerator of the diffusion-potential term. Equation 15, which involves the NP potential $\Phi_{\mathrm{NP}}$, has a term in the same location equal to $\left(1-2 t_{+}\right)$. This observation reveals an apparent discrepancy in the modified versions of Ohm's law provided by NP theory and OSM theory, which is readily resolved by recognizing that the two potentials have inconsistent thermodynamic definitions.

Equation 36 is on sounder thermodynamic footing than Equation 15 , because it has been established how the potential involved can be measured with a clearly defined probe (cf. the subsection concerning Thermodynamics of reference electrodes above). We might ask what, exactly, the NP voltage means from the standpoint of measurement.

NP theory is undergirded by an assumption of extreme dilution. This limit should be applied to the OSM equations to ensure the mutual consistency of properties before the two theories are compared. It has been asserted that the difference between $\Phi$ and $\Phi_{\mathrm{NP}}$ arises from differing definitions of transport properties within the NP and OSM theories. ${ }^{9}$ Actually this is not the case; it can be shown that the properties involved agree in the dilute limit as follows.

The ion diffusivities $D_{i}$ in NP theory can be identified as the highdilution limits of the Stefan-Maxwell coefficients $\mathscr{D}_{0 i}$ for ion/solvent interactions, i.e.,

$$
D_{+}=\lim _{c / c_{0} \ll 1} \mathscr{D}_{0+} \quad \text { and } \quad D_{-}=\lim _{c / c_{0} \ll 1} \mathscr{D}_{0-} .
$$

At high dilution the solution's mixing entropy is ideal, and the solvent volume fraction approaches unity, so

$$
\lim _{c / c_{0} \ll 1} \chi=1 \quad \text { and } \quad \lim _{c / c_{0} \ll 1} \bar{V}_{0} c_{\mathrm{T}}=1 .
$$

With these relationships and Equation 29, the salt diffusivities and cation transference numbers from the two theories are found to equate in the dilute-solution limit,

$$
D=\lim _{c / c_{0} \ll 1} \mathscr{D} \quad \text { and } \quad t_{+}=\lim _{c / c_{0} \ll 1} t_{+}^{0} .
$$

In the limit, these relations can be used to show that the ionic conductivity satisfies

$$
\lim _{c / c_{0} \ll 1} \frac{F^{2} z_{+}^{2} \nu_{+}^{2} c}{\nu R T \kappa}=\frac{t_{+}\left(1-t_{+}\right)}{D}+\frac{\nu_{+} \nu_{-}}{\nu} \lim _{c / c_{0} \ll 1} \frac{c}{c_{0} \mathscr{D}_{+-}} .
$$

Assuming that the concentration dependence of $\mathscr{D}_{+-}$is sufficiently weak that the second term on the right vanishes in the dilute limit, this agrees precisely with Equation 14.

Incorporation of Equations 37 through 40 into the OSM theory shows that the MacInnes equation for a solution with a cationreversible reference electrode can be written in the dilute limit as

$$
\frac{\vec{i}}{\kappa}=-\vec{\nabla} \Phi+\frac{R T \nu\left(1-t_{+}\right)}{F z_{+} \nu_{+}} \vec{\nabla} \ln c \quad \text { if } \quad \frac{c}{c_{0}} \ll 1 .
$$

By subtracting Equation 41 from Equation 15, and recognizing that the two potentials differ, one finds that the infinite-dilution limit of OSM theory yields a comparison

$$
\vec{\nabla} \Phi=\vec{\nabla} \Phi_{\mathrm{NP}}+\frac{R T}{F z_{+}} \vec{\nabla} \ln c .
$$

One can now see how ion electrochemical potentials must be defined in NP theory. Since $\vec{\nabla} \mu_{+}=F z_{+} \vec{\nabla} \Phi$ for the chosen reference electrode, the theories match if NP theory is recognized to be based on ion electrochemical potentials with constitutive laws

$$
\begin{aligned}
& \mu_{+}=\mu_{+}^{\ominus}+R T \ln c_{+}+F z_{+} \Phi_{\mathrm{NP}} \\
& \mu_{-}=\mu_{-}^{\ominus}+R T \ln c_{-}+F z_{-} \Phi_{\mathrm{NP}} .
\end{aligned}
$$

An electrolyte whose ions obey these constitutive laws is called an 'ideal electrolyte'. Thus the NP voltage is identified as the quasielectrostatic potential of an ideal electrolytic solution.

Observe that the solution-phase potential $\Phi_{\mathrm{NP}}$ is not a thermodynamic potential measured by a reference electrode, and is therefore somewhat distanced from an experimentally measurable voltage. The open-circuit potential (OCP) of a cell in which cations react should depend on cation chemical potentials, which incorporate the free energies of mixing, solvation, etc., rather than on a purely electrostatic quantity like $\Phi_{\mathrm{NP}}$.

To get experimental cell voltages from NP theory, one should use the values of $\Phi_{\mathrm{NP}}$ and the ion concentrations to compute electrochemical potentials through Equations 43. At a hypothetical electrode surface, an equilibrium expression like Equation 31 should be used to associate these chemical potentials with the voltage. If this reference electrode is reversible only to cations and implements their complete reduction, the potential measured in the laboratory for a dilute electrolyte will be governed by Equation 41 .

\section{Case Study for Lithium-Ion Battery Simulation}

This section illustrates the effect of using the NP voltage $\Phi_{\mathrm{NP}}$ instead of the reference-electrode voltage $\Phi$ during the modeling of a lithium-ion battery cell. A detailed description of the model and parameters used is provided by Bizeray et al. ${ }^{10}$

The lithium-ion battery model considered is the so-called pseudotwo dimensional (P2D) model coupled to a lumped thermal model. The partial differential equations involved were spatially discretized using Chebyshev orthogonal collocation and integrated over time using the MATLAB solver ode15s. The cell is assumed to be of 18650 type, consisting of a $\mathrm{LiCoO}_{2}$ cathode and a mesocarbon microbead (MCMB) anode, with an electrolyte comprising $1 \mathrm{M} \mathrm{LiPF}_{6}$ in PC:EC:DMC.

The P2D model was solved using the OSM MacInnes Equation 41 in the dilute limit (containing the $2\left(1-t_{+}\right)$term). The NP MacInnes Equation 15 was also used (with a $\left(1-2 t_{+}\right)$term); but relation 42 was neglected, i.e., $\Phi_{\mathrm{NP}}$ was taken to be the measured voltage instead of $\Phi$.

Figure 1a shows the evolution of the solution-phase potential drop across the lithium-ion cell using $\Phi$ and $\Phi_{\mathrm{NP}}$ during galvanostatic discharge at several $C$-rates. As expected because the lithium-ion transference number is close to $0.5\left(t_{+}=0.435\right)$, the use of the NP voltage $\Phi_{\mathrm{NP}}$ underestimates the solution-phase potential drop substantially at all rates because the magnitude of $\left(1-2 t_{+}\right)$is near zero, whereas $2\left(1-t_{+}\right)$is of order unity. As Figure 1b shows, however, this $30 \%$ error in solution-phase voltage drop does not largely impact the cell's terminal-to-terminal voltage, which is dominated by the open-circuit voltage associated with the cell reaction.

Although its effect on terminal voltage is small, the solution-phase potential drop within the electrode sandwich significantly affects heat generation associated with ion flow (ohmic) and interfacial reactions (kinetic). The $30 \%$ difference in solution-phase potential drop leads to significant differences in cell temperature rise during the discharge, especially at high C-rates, as Figure 1c shows. 

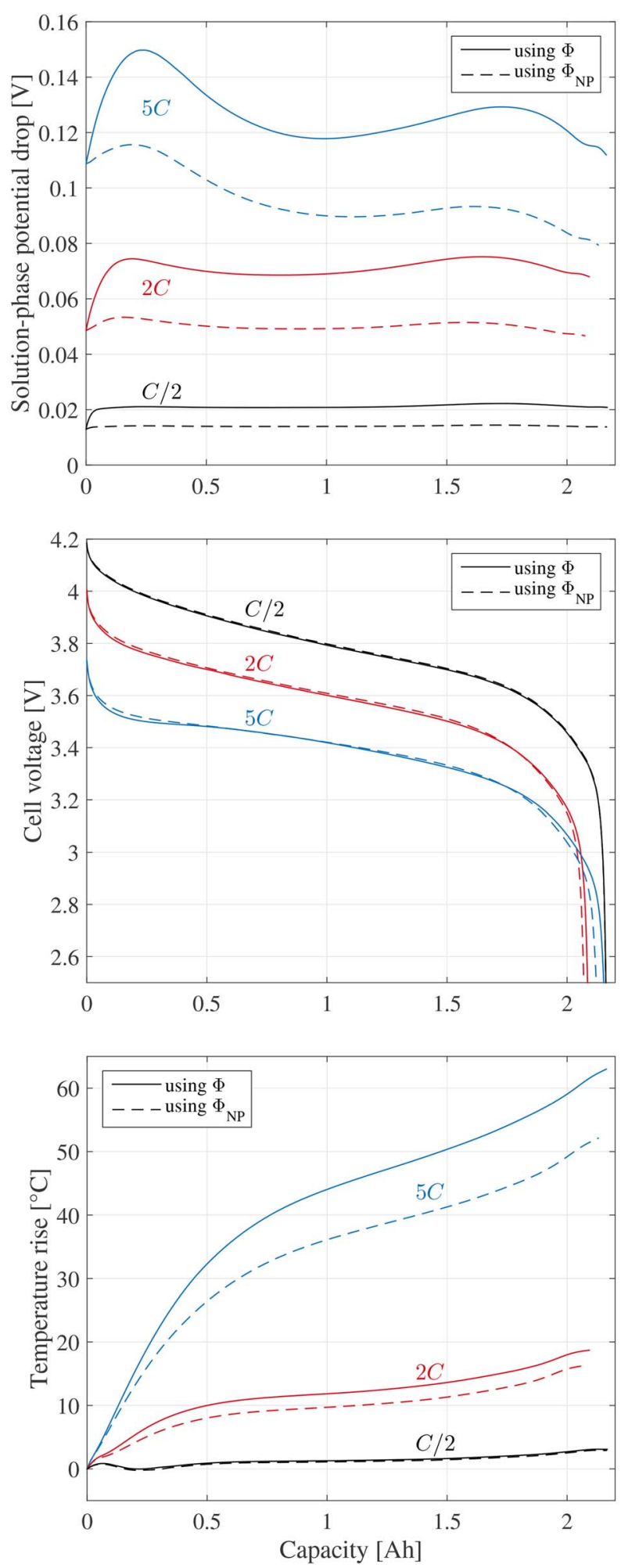

Figure 1. Evolution of (a) the solution-phase potential drop (anode-cathode) within a Li-ion cell, (b) the overall cell voltage, and (c) the cell bulk temperature, using the reference-electrode voltage $\Phi\left(\right.$ term $2\left(1-t_{+}\right)$in the MacInnes equation) and the NP voltage $\Phi_{\mathrm{NP}}$ (term $1-2 t_{+}$) during constant-current discharge at $C / 2,2 C$ and $5 C$.

In the range of higher state of charge (SOC), the maximum error in voltage does not exceed $30 \mathrm{mV}$ at any of the three discharge currents investigated. The maximum error in terminal-to-terminal voltage occurs at very low SOC because of the discrepancy in apparent cell capacity that results from changes in cell temperature.
Although the overall impact on cell terminal voltage seems small, the difference in internal battery states is not negligible, especially during high-current simulations. The use of an inconsistent solutionphase potential can induce large errors in local overpotential and heat generation, which are both vital for investigating battery degradation and thermal management.

\section{Conclusions}

A discrepancy between expressions for diffusion potential arising from Nernst-Planck theory and Onsager-Stefan-Maxwell theory has been resolved. In the dilute limit, the potentials in the two theories relate through Equation 42. The voltage across a dilute binary electrolyte as measured by a reference electrode where cations react, indicated by the symbol $\Phi$, is given by Equation 41, which has a prefactor of $2\left(1-t_{+}\right)$in the diffusion-potential term. Equation 15 , which has a prefactor of $\left(1-2 t_{+}\right)$instead, involves an idealized quasi-electrostatic potential, $\Phi_{\mathrm{NP}}$.

NP theory employs what one could call a 'solution-phase' potential, commonly used by the community to describe the internal states of semiconductors, space-charge regions in supercapacitors, and solid ion conductors. The fact remains that the potential $\Phi$ is directly connected to the measurable voltage of a cell, whereas $\Phi_{\mathrm{NP}}$ is more abstract. Although neglecting the distinction between these voltages may have only a small impact on the simulated terminal-to-terminal voltage of a device such as a lithium-ion battery, it has a large effect on internal state variables such as overpotential and temperature. It is therefore crucial to consider the thermodynamic definition of potential when modeling electrochemical devices for design and estimation purposes.

\section{Acknowledgments}

This work was sponsored by the U.S. Department of Energy Office of Energy Efficiency and Renewable Energy, Vehicle Technologies Office and Advanced Battery Material Research Program, under contract DE-EE-00006821. A.M.B. was supported by Samsung Electronics Co. Ltd. through a Global Research Outreach program in collaboration with the Samsung Advanced Institute of Technology.

\section{References}

1. A. M. Ramos, On the well-posedness of a mathematical model for lithium-ion batteries, Appl. Math. Modell., 40, 115 (2016).

2. P. M. Gomadam, J. W. Weidner, R. A. Dougal, and R. E. White, Mathematical modeling of lithium-ion and nickel battery systems, J. Power Sources, 110(2), 267 (2002).

3. K. Smith and C-Y. Wang, Power and thermal characterization of a lithium-ion battery pack for hybrid-electric vehicles, J. Power Sources, 160(1), 662 (2006).

4. K. Smith and C-Y. Wang, Solid-state diffusion limitations on pulse operation of a lithium ion cell for hybrid electric vehicles, J. Power Sources, 161(1), 628 (2006).

5. K. Smith, C. D. Rahn, and C-Y. Wang, Control oriented 1D electrochemical model of lithium ion battery, Energy Convers. Manage., 48(9), 256 (2007).

6. N. Chaturvedi, R. Klein, J. Christensen, J. Ahmed, and A. Kojic, Algorithms for Advanced Battery-Management Systems, IEEE Control Syst. Mag., 30(3), 49 (2010).

7. G-H. Kim, K. Smith, J. Ireland, and A. Pesaran, Fail-safe design for large capacity lithium-ion battery systems, J. Power Sources, 210, 243 (2012).

8. M. W. Verbrugge and P. Liu, Microstructural analysis and mathematical modeling of electric double-layer supercapacitors, J. Electrochem. Soc., 152(5), D79 (2005).

9. G. Richardson, G. Denuault, and C. P. Please, Multiscale modeling and analysis of lithium-ion battery charge and discharge, J. Eng. Math., 72, 41 (2012).

10. A. M. Bizeray, S. Zhao, S. R. Duncan, and D. A. Howey, Lithium-ion battery thermalelectrochemical model-based state estimation using orthogonal collocation and a modified extended Kalman filter, J. Power Sources, 296, 400 (2015).

11. J. Newman and K. E. Thomas-Alyea, Electrochemical Systems, 3rd Edition, John Wiley \& Sons, Inc., Hoboken, New Jersey (2004).

12. M. Planck, Ueber die Potentialdifferenz zwischen zwei verdünnten Lösungen binärer Electrolyte, Ann. Phys. Chem., 40, 561 (1890).

13. L. Onsager, Theories and problems of liquid diffusion, Ann. N. Y. Acad. Sci., 46, 241 (1945).

14. D. A. MacInnes, The Principles of Electrochemistry, 2nd Ed., Dover Publications, Inc., New York, (1961).

15. W. H. Smyrl and J. Newman, Potentials of cells with liquid junctions, J. Phys. Chem., 72, 4660 (1968) 
16. S. R. de Groot, Thermodynamics of Irreversible Processes, Interscience Publishers, New York, (1951).

17. J. Newman, Effect of ionic migration on limiting currents, Ind. Eng. Chem. Fundam., 5, $525(1966)$

18. J. Newman, "Transport Processes in Electrolytic Solutions," in C.W. Tobias, ed., Advances in Electrochemistry and Electrochemical Engineering, vol. 5, Interscience Publishers, J. Wiley and Sons, New York, 1967.

19. J. Newman and T. W. Chapman, Restricted diffusion in binary solutions, AIChE J., 19, 343 (1973).

20. J. O. Hirschfelder, C. F. Curtiss, and R. B. Bird, Molecular Theory of Gases and Liquids, John Wiley \& Sons, New York, (1954).
21. E. N. Lightfoot, E. L. Cussler Jr., and R. L. Rettig, Applicability of the StefanMaxwell equations to multicomponent diffusion in liquids, AIChE J., 8, 708 (1962).

22. J. Liu and C. W. Monroe, Solute-volume effects in electrolyte transport, Electrochim. Acta, 135, 447 (2014)

23. J. Liu and C. W. Monroe, On the characterization of battery electrolytes with polarization cells, Electrochim. Acta, 167, 357 (2015)

24. E. A. Guggenheim, Thermodynamics: an Advanced Treatment for Chemists and Physicists, 5th Ed., North-Holland Publishing Company, Amsterdam (1967).

25. T. W. Chapman and J. Newman, A Compilation of Selected Thermodynamic and Transport Properties of Binary Electrolytes in Aqueous Solution, Technical Report UCRL-17767, University of California, Lawrence Radiation Laboratory (1968). 